\title{
The Mellow Years?: Neural Basis of Improving Emotional Stability over Age
}

\author{
Leanne M. Williams, ${ }^{1,2}$ Kerri J. Brown, ${ }^{1}$ Donna Palmer, ${ }^{1,4}$ Belinda J. Liddell, ${ }^{1,4}$ Andrew H. Kemp, ${ }^{1,2}$ Gloria Olivieri, ${ }^{1,3}$ \\ Anthony Peduto, ${ }^{1,3}$ and Evian Gordon ${ }^{1,2,5}$ \\ ${ }^{1}$ Brain Dynamics Centre, Westmead Millenium Institute and Western Clinical School, University of Sydney, Westmead Hospital, ${ }^{2}$ Psychological Medicine, \\ Western Clinical School, University of Sydney, and ${ }^{3}$ Magnetic Resonance Imaging Unit, Department of Radiology, Westmead Hospital, Westmead, Sydney, \\ New South Wales, 2145, Australia, ${ }^{4}$ School of Psychology, University of Sydney, Camperdown, Sydney, New South Wales, 2006, Australia, and ${ }^{5}$ Brain \\ Resource International Database, Brain Resource Company, Ultimo, Sydney, New South Wales, 2007, Australia
}

Contrary to the pervasive negative stereotypes of human aging, emotional functions may improve with advancing age. However, the brain mechanisms underlying changes in emotional function over age remain unknown. Here, we demonstrate that emotional stability improves linearly over seven decades (12-79 years) of the human lifespan. We used both functional magnetic resonance imaging and event-related potential recording to examine the neural basis of this improvement. With these multimodal techniques, we show that better stability is predicted by a shift toward greater medial prefrontal control over negative emotional input associated with increased activity later in the processing sequence (beyond $200 \mathrm{~ms}$ after stimulus) and less control over positive input, related to a decrease in early activity (within $150 \mathrm{~ms}$ ). This shift was independent from gray matter loss, indexed by structural magnetic resonance data. We propose an integrative model in which accumulated life experience and the motivation for meaning over acquisition in older age contribute to plasticity of medial prefrontal systems, achieving a greater selective control over emotional functions.

Key words: functional brain imaging; medial prefrontal cortex; amygdala; basal ganglia; emotion; human aging

\section{Introduction}

Human aging is associated with pervasive negative stereotypes of declining sensory and cognitive integrity. Although this view is supported by evidence of reductions in memory and executive planning with advancing age (Craik and Salthouse, 2000), less attention has been given to functions that improve. Contrary to the negative stereotypes, emotional wellbeing may increase with normal aging. Behavioral studies have reported less negativity, an easing of emotional intensity, and a reduction in trait neuroticism, reflecting better emotional stability, with increasing age (George et al., 1988; Whittington and Huppert, 1998; Lachman and Bertrand, 2001; Mroczek and Spiro, 2003; Phillips and Allen, 2004). Notably, older adults also show a shift in the ratio of positive-to-negative emotion, with a reduction in the experience, memory, and recognition of negative emotion, but an increase for positive emotion (Malatesta et al., 1987; Moreno et al., 1993; Gross et al., 1997; Calder et al., 2003).

Several models have been put forward to account for agerelated changes in emotional function. Those focused on envi-

\footnotetext{
Received Dec. 20, 2005; revised April 18, 2006; accepted April 24, 2006.

This work was supported in part by Australian Research Council Grants DP0452237 and DP0345481 (to L.M.W., with E.G. and K.J.B.). L.M.W. was supported by a Pfizer Senior Research Fellowship. A.H.K. was supported by a National Health and Medical Research Council Peter Doherty Fellowship. We thank the Brain Resource International Database (under the auspices of the Brain Resource Company) for data acquisition support.

Correspondence should be addressed to Assoc. Prof. Leanne M. Williams, Brain Dynamics Centre, Westmead Millenium Institute, Westmead Hospital, Westmead, New South Wales, 2145, Australia. E-mail: lea@psych.usyd.edu.au.

D0I:10.1523/JNEUROSC1.0022-06.2006

Copyright $\odot 2006$ Society for Neuroscience $\quad$ 0270-6474/06/266422-09\$15.00/0
}

ronmental influences propose that retirement may be a key factor in reducing daily stress, and thus negativity, in older age (Carstensen and Lockenhoff, 2003). However, these influences would not readily account for a selective shift in positive versus negative emotion. Others suggest that selective changes may reflect differential rates of normal age-related atrophy in the brain structures associated with processing positive and negative emotion (Calder et al., 2003), yet atrophy cannot readily explain improvements in emotion recognition and stability. Social cognitive researchers propose that these improvements reflect a shift in motivational priorities over age, from knowledge acquisition and competition to the pursuit of emotional satisfaction, attributable to the increasing awareness of a limited lifespan. From this view, attention and memory increase with age for positive information, but decrease for negative, to benefit emotional stability and regulation of social behavior (Carstensen and Lockenhoff, 2003; Oschsner et al., 2004). Similarly, age-related reductions in neuroticism have been interpreted as an increase in maturity and personal adjustment in the sense of being less emotionally volatile and more attuned to social roles (Helson and Wink, 1987; Roberts and Caspi, 2003). Although the social cognitive account is able to explain both improvements and selective changes in emotion processing over age, the underlying neural mechanisms remain unknown.

Lack of research into neural mechanisms of better emotional function with age may reflect a presumption that evolutionary selection has not acted on the postproductive years. However, a significant number of postproductive adults present among our 


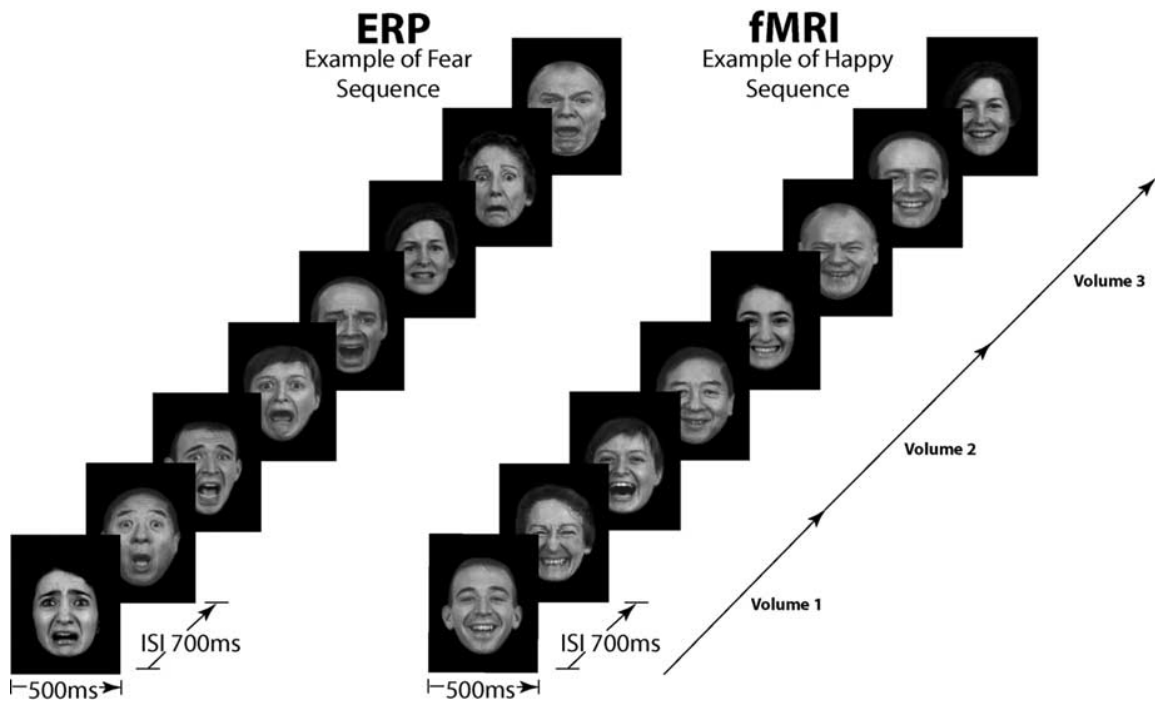

Figure 1. Summary of the design of the behavioral facial emotion perception task used with identical stimuli and parameters in functional MRI and ERP testing sessions. Shown here is an example sequence of fearful faces used in ERP testing and an example sequence of happy faces used in functional MRI testing.

human ancestors may have contributed to gene survival via important social roles (Carstensen and Lockenhoff, 2003). In this study, we focused on the medial prefrontal cortex (MPFC) implicated in the governance of emotional functions (Damasio, 1996) and the subcortical circuits associated with emotion processing (Morris et al., 1996; Phan et al., 2002). We used complementary event-related potential (ERP) and functional magnetic resonance imaging (fMRI) techniques with a large group of healthy individuals spanning a wider spectrum of the lifespan (12-79 years) than studied previously. Using these measures, we determined whether changes in brain function over these seven decades reflect a selective shift in processing standardized facial signals of positive and negative emotion. We examined whether this shift predicts corresponding changes in emotion recognition and emotional stability, assessed in terms of neuroticism. Brain structure was also examined to consider the potential contribution of differential changes in brain atrophy.

\section{Materials and Methods}

Participants. Participants comprised 242 healthy individuals (mean \pm SD age, $34.79 \pm 16.49$ years), with an equivalent distribution of sexes (122 males, 120 females), recruited in collaboration with the Brain Resource International Database (Gordon et al., 2005). These subjects were those who completed testing sessions from an initial pool of 250 volunteers. Subjects were screened using strict and standardized exclusion criteria: history of mental illness, brain injury, neurological disorder or other serious medical condition, and history of substance abuse. The ethnic breakdown of the sample was $96.2 \%$ Caucasian European and $3.8 \%$ Asian background.

For contrast analyses, subjects were grouped into four age bands, selected on several criteria. They represented points of change in the lifespan (maturation, young to middle adulthood, and aging), which were confirmed as being points of significant change in brain function data. They also represented a quartile split of the age distribution and were therefore of approximately equal size: teens ( $12-19$ years; $n=60$; mean, 16.1 years), young (20-29 years; $n=59$; mean, 24.8 years), middle ( $30-49$ years; $n=63$; mean, 39.7 years), and older ( $50-79$ years; $n=60$; mean, 59.3 years).

Participants provided written informed consent in accordance with national health and medical research council guidelines.

Behavioral assessment and analysis. We assessed emotional stability using a self-report index of neuroticism from the NEO-Five Factor In- ventory, with a score range of $0-60$ (Costa and McCrae, 1992). Regression analysis was used to determine whether age predicted recognition accuracy and neuroticism scores. A priori contrasts provided complementary comparisons between age groups.

After brain function testing, emotion recognition accuracy for each of the face stimuli was assessed using a multiple-option procedure. Subjects were shown the eight different individuals depicting expressions of fear, happiness, and neutral used in fMRI and ERP testing interspersed with "distractor" expressions depicting anger, disgust, and sadness, with six presentations of each in a randomized sequence. They also rated the perceived intensity of each expression on a scale of 1 (extremely mild) to 5 (extremely intense). Emotion recognition and intensity was also analyzed with regression analyses and complementary contrast between the four age groups.

Behavioral task. Facial expressions of emotion provide salient exemplars of negative (fearful expressions conveying potential threat) and positive (expressions of happiness, conveying positive intent and pleasure) emotion, relevant to human interaction. Face stimuli displaying evoked expressions of fear and happiness, as well as neutral control expressions, were selected from a standardized set of facial emotion stimuli (Mather et al., 2004) on the basis that they had been rated as the most accurate representations of each respective emotion. These stimuli were equated in terms of size and luminance. Static expression stimuli are the most commonly used in neuroimaging studies, and we used such stimuli to enable interpretation of age effects in the context of previous findings regarding the neural correlates of emotion processing. Second, given that ERPs and fMRI have different temporal resolutions, the use of static expressions ensured that the stimulation eliciting activity in each case was standardized. Static facial expressions of emotion, presented for as briefly as $500 \mathrm{~ms}$, are sufficient to elicit the subjective experience of emotion (reflected in changes in skin conductance arousal), feasibly via a process of "contagion" (Wild et al., 2001; Williams et al., 2005).

During ERP recording, blocks containing eight randomized stimuli depicting fear, happy, or neutral were presented, with blocks in pseudorandom order and stimuli randomized within blocks. There were therefore four repeat blocks for each expression, making a total of 32 stimuli per expression. Stimuli were presented for $500 \mathrm{~ms}$, with an interstimulus interval (ISI) of $700 \mathrm{~ms}$. For fMRI recordings, the same facial emotion stimuli were presented within an equivalent behavioral task using a projector (ProX, Multiverse; Sanyo, Gallenkamp, UK) and mirror system. Stimuli were again presented for $500 \mathrm{~ms}$, with an ISI of $700 \mathrm{~ms}$. A summary of the equivalent behavioral tasks used in ERP and fMRI recordings is shown in Figure 1. For each recording session, subjects were instructed to attend to the faces in preparation for the posttesting behavioral assessments.

Previously, concurrent recording of skin conductance responses (SCRs) with functional MRI has shown that facial emotion stimuli elicit changes in subjective emotional experience (Williams et al., 2001, 2005), consistent with the notion of contagion (Wild et al., 2001). In this study, concurrent SCR recording provided confirmation of changes in emotional arousal associated with emotion perception: SCRs were enhanced for both fear $(t=2.22 ; p=0.035)$ and happiness $(t=3.72 ; p=0.001)$ relative to neutral (for details of SCR method, see Williams et al., 2001, 2005).

ERP acquisition and analysis. Participants were seated in a sound and light attenuated room, with temperature controlled at $24^{\circ} \mathrm{C}$. Data were acquired continuously at $500 \mathrm{~Hz}$ with skin resistance of $<5 \mathrm{k} \Omega$ from using a Quikcap and NuAmps system according to the international 10-20 electrode system. The focal sites of interest for this study were those implicated in face processing: medial fronto-central $(\mathrm{Fz}, \mathrm{Cz})$ and 
temporal (left, T5; right, T6) and visual occipital (left, O1; right, O2). Data were recorded relative to the virtual ground and referenced off-line to linked mastoids. Eye movement correction was undertaken off-line.

ERPs were scored following a well established procedure (Williams et al., 2006a). Single-trial ERP epochs (responses to each stimulus) were filtered with a low-pass Tukey's (cosine taper) filter function that attenuated frequencies above $25 \mathrm{~Hz}$ and averaged for fear and happiness relative to neutral. Peak components were identified within latency windows established in a previous study using a large sample of healthy controls $(n=219)$ (Williams et al., 2006a) and validated by visual inspection of the ERP waveforms at each recording site for 180 individual subjects in this study. These latency windows were also similar to those used in previous ERP studies of facial emotion perception (Eimer and Holmes, 2002; Ashley et al., 2004). At the medial frontal (Fz, Cz) and temporo-occipital ( $55, \mathrm{~T} 6, \mathrm{O} 1, \mathrm{O} 2)$ regions of focal interest, the following latency windows [and components ( $\mathrm{P}$, positivity; $\mathrm{N}$, negativity)] were identified: medial, 40-120 ms (P80), 80-150 ms (N120), 120-220 ms [vertex positive potential (VPP)], 180-280 $\mathrm{ms}$ (N200), and 280-450 ms (P300); temporo-occipital, 80-180 ms (P120), 120-220 ms (N170), $180-290 \mathrm{~ms}$ (P230), and 230-350 ms (N250). Temporo-occipital components represented the polarity reversal of those at medial sites, for the corresponding time windows. Each component was quantified in terms of peak-to-peak amplitude.

Regression analyses were used to examine changes in ERP components over age as a continuous variable. Complementary repeated-measures multivariate ANOVAs (MANOVAs) were used to analyze changes over each age group as a between-group factor with emotion (fear/happy vs neutral) as the within-subjects factor, followed by planned contrasts at a corrected significance level of $p<0.01$.

Functional MRI acquisition and analysis. Functional MRI was undertaken with a matched subset of one-third $(n=80)$ of subjects from those used in behavioral assessments and ERP testing. After the exclusion of two subjects attributable to excessive movement in fMRI testing, the age groups were teens ( $12-19$ years; $n=18)$, young (20-29 years; $n=24)$, middle ( $30-49$ years; $n=18)$, and older $(50-79$ years; $n=17)$. Imaging was performed on a $1.5 \mathrm{~T}$ Siemens (Munich, Germany) Vision Plus scanner using an echo echo-planar protocol. A total of 90 functional $\mathrm{T} 2{ }^{\star}$-weighted volumes were acquired, comprising 15 noncontiguous slices parallel to the intercommissural (anterior commissure-posterior commissure) line, with $6.6 \mathrm{~mm}$ thickness, repetition time (TR) of $3.3 \mathrm{~s}$, echo time (TE) of $40 \mathrm{~ms}$, flip angle of $90^{\circ}$, field of view of $24 \times 24 \mathrm{~cm}^{2}$, and matrix size of $128 \times 128$. Because of the slower temporal resolution of fMRI (relative to ERPs), three volumes per block (of eight stimuli) were acquired. Three initial "dummy" volumes were also acquired to ensure blood oxygen level-dependent (BOLD) saturation. We demonstrated the ability of this imaging protocol to elicit robust activity in the amygdala (Williams et al., 2006b). Observed signal-to-noise ratio (SNR) values, calculated according to LaBar et al. (2001), were shown to well exceed the expected minimum SNR values reported by Parrish et al. (2000) for both healthy and clinical subject groups.

Preprocessing and statistical analysis was implemented within the statistical parametric mapping software package SPM2 (http://www.fil. ion.ucl.ac.uk/spm/software). For each subject, functional images were first corrected for susceptibility-by-movement artifacts and then realigned to the first volume of the time series. Realigned images were spatially normalized into the standard stereotactic space of the Montreal Neurologic Institute (MNI) and smoothed using a Gaussian kernel [fullwidth half-maximum (FWHM), $8 \mathrm{~mm}$ ] to minimize anatomical differences. Experimental conditions (fear/happy vs neutral) were modeled using a canonical hemodynamic response function with temporal derivative, and a high-pass filter was applied to remove low-frequency fluctuations in the BOLD signal.

A priori search regions of interest (ROIs) were defined by standardized masks based on neuroanatomical divisions and defined by the automated anatomical labeling (AAL) protocol (Tzourio-Mazoyer et al., 2002): for medial prefrontal cortex (encompassing the anterior cingulate cortex, BA24/32, and medial orbital to superior frontal structures, extending to BA9/10), amygdala, and basal ganglia (encompassing caudate, putamen, and nucleus accumbens).
Functional maps were constructed for the activated voxels within each ROI for the contrasts of fear and happiness relative to neutral. We used an $\alpha$ level of $p<0.05$ (with small volume correction) and a spatial extent of at least five voxels per cluster. Age was first examined as a continuous variable in regression analyses for fear and happiness. ANOVA was then used to examine emotion by age group, and significant effects involving age were explored using a priori $t$ test contrasts.

Structural MRI acquisition and analysis. A high-resolution structural image was undertaken for each subject in the same session as fMRI, using a single T1-weighted volumetric magnetization-prepared rapidacquisition gradient echo structural scan. Images were obtained in the sagittal plane, with the following scan parameters: TR of $9.7 \mathrm{~ms}$, TE of 4 $\mathrm{ms}$, inversion time of $200 \mathrm{~ms}$, and flip angle of $12^{\circ}$. A total of 180 contiguous $1 \mathrm{~mm}$ slices were acquired with a $256 \times 256$ matrix with an in-plane resolution of $1 \times 1 \mathrm{~mm}$, resulting in isotropic voxels.

We undertook voxel-based morphometry using SPM2 to quantify gray matter volume for the same regions of interest used in the fMRI analyses (Ashburner and Friston, 2000; Good et al., 2001). Images were first spatially normalized by transforming each brain into a standardized stereotactic space based on the MNI ICBM 152 template. Normalized images were resliced with $1.5 \times 1.5 \times 1.5 \mathrm{~mm}$ voxels, then segmented into gray matter, white matter, and CSF probability maps, and stripped of extracerebral voxels. These images were smoothed with a Gaussian kernel (FWHM of $12 \mathrm{~mm}$ ) before volume calculation. The preprocessed gray matter images were then automatically parcellated into the AAL (Tzourio-Mazoyer et al., 2002) regions of interest (MPFC, amygdala, and basal ganglia) used in fMRI analyses. Regression analyses were then undertaken to determine whether age predicted changes in gray matter in these regions, followed by contrasts to examine significant effects.

Behavior and brain function relationships. Regression analyses were used to examine whether changes in brain function over age, in response to fear and happiness assessed using the fMRI and ERP measures, predicted changes in the neuroticism measure of emotional stability.

\section{Results}

Regression analyses and complementary contrasts examining age effects for behavior and brain function are reported in turn for each measure in relation to the hypotheses of the study. (The question of whether age-related changes in ERP and fMRI responses to facial emotion stimuli interact with sex is the subject of an independent study, which also addresses the role of automatic versus controlled processing of emotion. This study has shown that decreases in frontal activity for happiness and increases in frontal activity for fear are present for both males and females.

\section{Changes in neuroticism over age}

Consistent with our predictions, level of neuroticism showed a significant and linear decline over $12-79$ years of age $(F=17.62$; $p<0.0001$ ), reflecting increasing emotional stability as a function of age (Fig. 2). Contrasts confirmed that neuroticism was greatest in the teens (12-19 years) and least in older years (50-79 years), with significant $(p<0.01)$ differences between all age groups, excepting young and middle adulthood.

\section{Changes in emotion recognition over age}

Our predictions were also supported by opposing patterns of emotion recognition accuracy for fear and happiness. Whereas age predicted a decline in recognition accuracy for fear $(F=$ $17.80 ; p<0.001)$, it predicted an increase in accuracy for happiness $(F=15.18$; $p<0.0001)$ (Fig. 3). Contrasts confirmed that younger age groups were significantly $(p<0.0001)$ more accurate than older groups for fear, whereas the youngest (12-19 years) group was significantly less accurate than the three older age groups for happiness (Fig. 3). The perceived intensity of these expressions, conversely, showed a common linear attenuation as a function of increasing age for both fear $(F=9.31 ; p<0.001)$ 


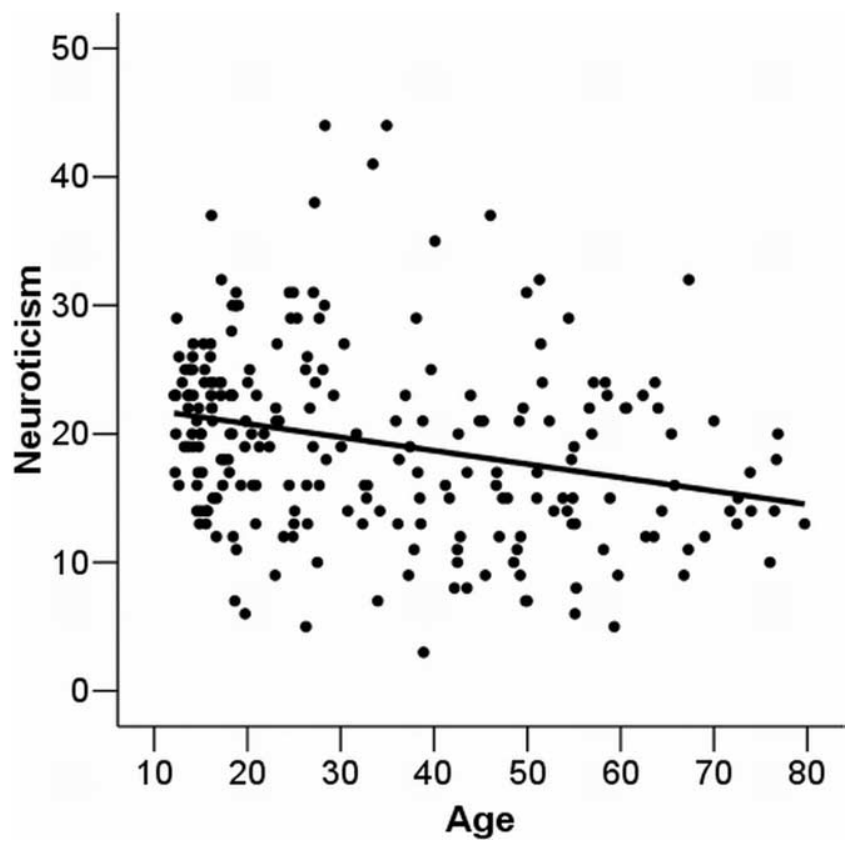

Figure 2. Scatter plot with regression line depicting the significant decline over age (in years) in level of neuroticism. As an index of emotional stability, the decline in neuroticism reflects better emotional stability with older age.
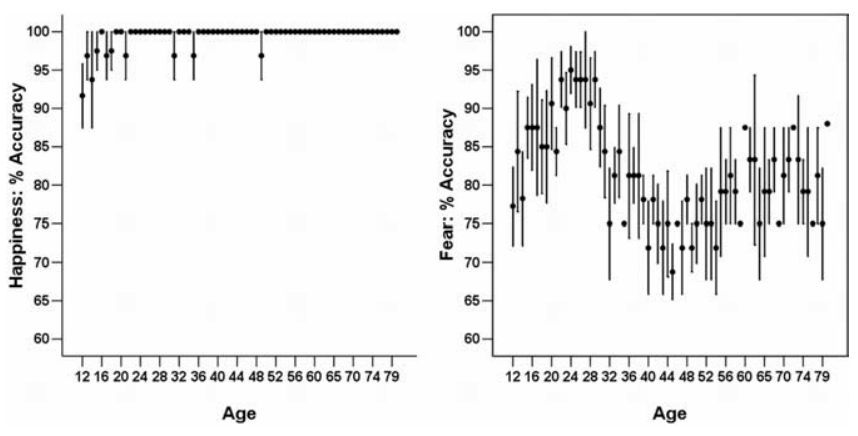

Figure 3. Error bar plot depicting changes over age (in years) for accuracy of recognition (as a percentage) for facial expressions of fear (right) and happiness (left). Given that accuracy was determined from eight repeats of each emotion, the range of possible percentage accuracy values was $12.5-100 \%$. Error bars show SE.

and happiness $(F=19.08 ; p<0.0001)$, confirmed in pairwise contrasts of age groups $(p<0.001)$.

\section{Changes in fMRI-recorded brain function over age}

Behavioral differences were echoed in distinctive profiles of underlying brain function over age for fMRI data. Regression analyses showed that increasing age was associated with a linear decrease in responses to happiness $(F=25.40$; $p<0.0001$; MNI coordinates for cluster with most significant effect, $x=-18, y=$ $22, z=54)$ but a linear increase in MPFC responses to fear $(F=$ $12.25 ; p<0.0001 ;$ MNI coordinates for cluster with most significant effect, $x=-14, y=36, z=42$ ) (Fig. 4). In contrast, regressions for the subcortical amygdala and basal ganglia were not significant.

Complementary ANOVA revealed a significant interaction between age and activation in the MPFC for happiness versus fear (Table 1). Consistent with regression analyses, MPFC activation was lower in older compared with younger groups for happiness
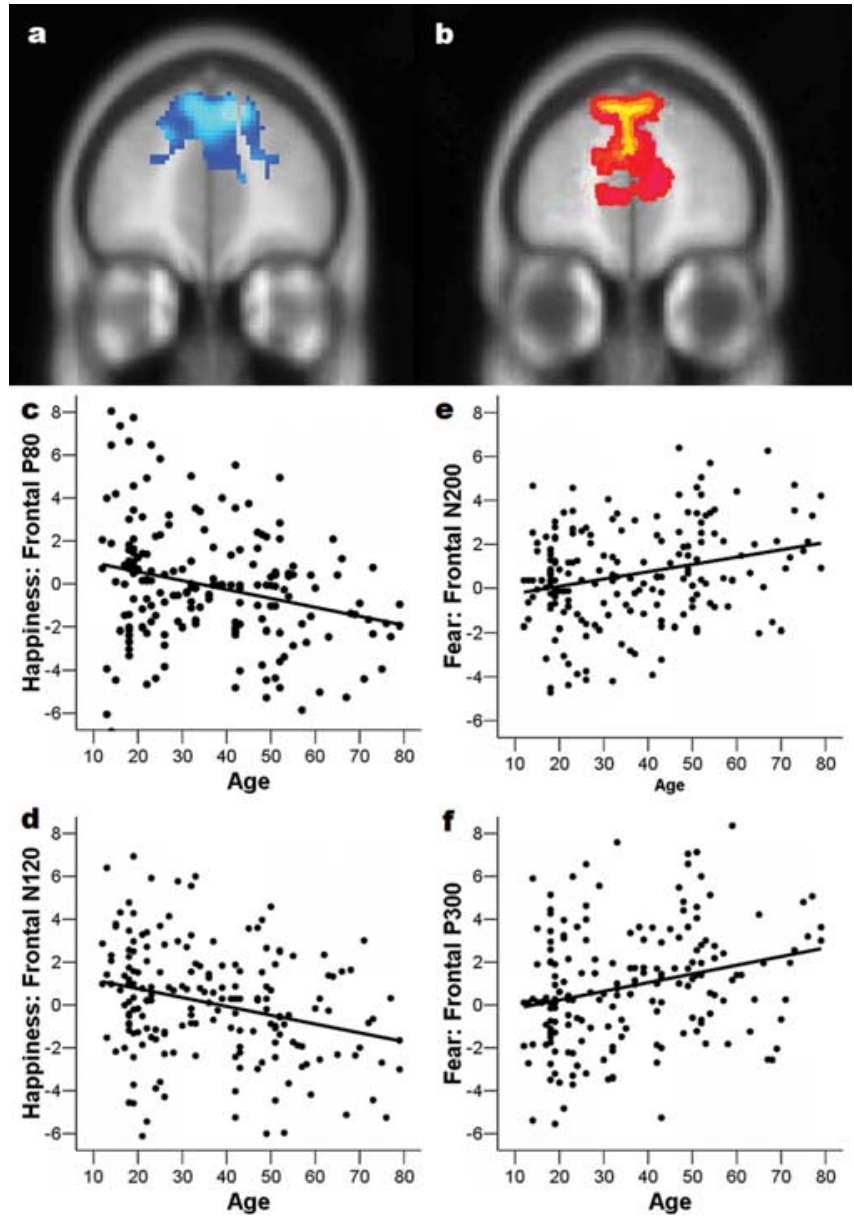

Figure 4. This figure illustrates the regression analyses for brain function data. $\boldsymbol{a}, \boldsymbol{b}$, For functional MRI, age has a negative association with activation in the medial prefrontal cortex for happiness $(\boldsymbol{a})$, shown in blue, but a positive association with activation in this region for fear $(\boldsymbol{b})$, shown in red. $\boldsymbol{c}, \boldsymbol{d}$, For ERPs, age has a corresponding negative association with early ERPs (40-150 ms) for happiness, shown in the scatter plot for the frontal P80 (c) and N120 (d).e, $\boldsymbol{f}$, In contrast, age has a corresponding positive association with later ERPs (180 - $450 \mathrm{~ms})$ for fear, shown in the scatter plot for the frontal N200 (e) and P300 (f).

but greater in older compared with younger groups for fear, reflected in significant contrasts (Table 1, Fig. 5).

There was also a significant interaction between age and responses to happiness versus fear for the subcortical basal ganglia and trend level interaction for the amygdala (Table 1). Contrasts showed that these interactions were attributable to a different and nonlinear profile of change, consistent with the nonsignificant linear regression results. For happiness, activation in both the amygdala and caudate region of the basal ganglia was greater in the teen and young adult (12-29 years) compared with middle adult and older (40-79 years) age groups (Table 1, Fig. 6). For fear, conversely, responses in the amygdala and caudate were greater in the young adult and middle (20-49 years) compared with younger (12-19 years) and older (50-79 years) age groups (Table 1, Fig. 6). Thus, the overlapping period of 20-29 years was the one of greatest subcortical activity for both happiness and fear.

\section{Changes in ERPs over age}

Regression analyses for ERP data provided convergent support for opposing patterns of change over age across the medial frontal region in response to happiness versus fear. There was also an 
additional dissociation in the timing of these changes over age. Advancing age was associated with a linear decrease in early ERPs (40-150 ms) over the medial frontocentral sites for happiness [the P80 $(F=$ $14.18 ; p<0.0001)$ and N120 $(F=15.91$; $p<0.0001)$ ] but an increase in later ERPs (180-450 ms) over the same frontocentral sites for fear [the N200 $(F=14.27$; $p<0.0001)$ and P300 ( $F=13.89 ; p<$ $0.0001)$ ] (Fig. 4). In contrast, age was not found to impact the temporo-occipital N170 and concomitant medial frontal VPP components, which reflect structural encoding of faces $\sim 120-220 \mathrm{~ms}$ after stimulus (Jeffreys, 1989; Streit et al., 2000). Null results for these components indicate that encoding of face stimuli was comparatively preserved across age for both happiness and fear and that emotion effects were not attributable simply to the differential effects of perceptual processing. Similarly, age did not impact the temporooccipital P230 and N250 components, indicating that age changes were localized to the frontal region.

Complementary support for the dissociation of early ERP components for happiness versus late components for fear was revealed by MANOVA and significant $(p<0.05)$ interactions between age and these components. Contrasts showed that older and middle adult age groups had reduced responses compared with younger adult and teen age groups for early positivity (P80) and subsequent negativity (N120) at the corrected level of $p<0.01$. For fear, older and middle adult age groups showed greater responses than younger adult and teen age groups for later negativity (N200) and subsequent positivity (P300) at $p<0.01$ (Fig. 5). In contrast, age did not interact with the N170/VPP encoding or temporo-occipital P230/N250 components, consistent with regression analyses.

\section{Changes in brain structure over age}

For structural MRI data, regression showed that only MPFC gray matter volume declined significantly as a function of age (left MPFC, $F=18.91$; right MPFC, $F=12.05 ; p<0.0001$ ). Complementary pairwise contrasts confirmed that older age groups had significantly less MPFC gray matter than younger ones $(p<$ $0.0001)$. In contrast, there was comparative preservation of the amygdala (left, $F=1.07, p=0.57$; right, $F=2.97, p=0.35$ ) and basal ganglia caudate region (left, $F=2.16, p=0.19$; right, $F=$ $1.04, p=0.77)$. The loss of MPFC gray matter was found to predict only the decline in fear recognition $(F=4.17 ; p<0.05)$ but not functional changes in this region or neuroticism.

\section{Prediction of age-related behavioral changes by brain function changes}

Importantly, the shift over age in underlying medial prefrontal brain systems for processing happiness versus fear accounted for better emotional stability. Level of neuroticism was predicted by the decreasing MPFC responses to happiness $(F=4.33 ; p<0.05)$ and increasing MPFC responses to fear $(F=4.16 ; p<0.05)$. Similarly, it was predicted by the decrease in the earlier N120 activity for happiness $(F=6.21 ; p<0.05)$ and by the increase in the later P300 component elicited to fear $(F=5.74 ; p<0.05)$. The age-related fMRI changes in subcortical activity, in contrast, did not predict the improvement in emotional stability, highlighting the specific role of the medial prefrontal region. Changes in medial prefrontal function were also found to predict the attenuation in ratings of perceived emotional intensity. The reduction in perceived fear intensity was predicted by the increase in MPFC activation to fear in $\mathrm{fMRI}$ data $(F=5.02 ; p<0.05)$ and corresponding increase in the medial fronto-central P300 $(F=$ 4.99; $p<0.05$ ), whereas the reduction in perceived intensity of happiness was predicted by decreasing MPFC activation in this region $(F=6.83 ; p<0.01)$ and the decrease in both the $\mathrm{P} 80(F=$ $5.53 ; p<0.05)$ and N120 $(F=4.92 ; p<0.05)$ components for happiness.

\section{Discussion}

These findings provide new evidence that emotional wellbeing improves over seven decades of the human lifespan, reflected in better emotional stability and an easing of perceived emotional intensity as a function of age. Improvements in emotional stability were predicted by an adaptive shift toward greater controlled processing of negative emotion, with a corresponding reduction for positive emotion, involving the medial prefrontal cortex. We propose an integrative model in which the change in motivational priorities and accumulated life experience of older age bring greater selectivity in the perception of positive versus negative emotion and impact neural plasticity to produce a corresponding shift in underlying medial prefrontal systems, which contributes to better emotional stability.

The shift in responses to positive versus negative emotion over 


\section{Decreasing early responses to happiness.}

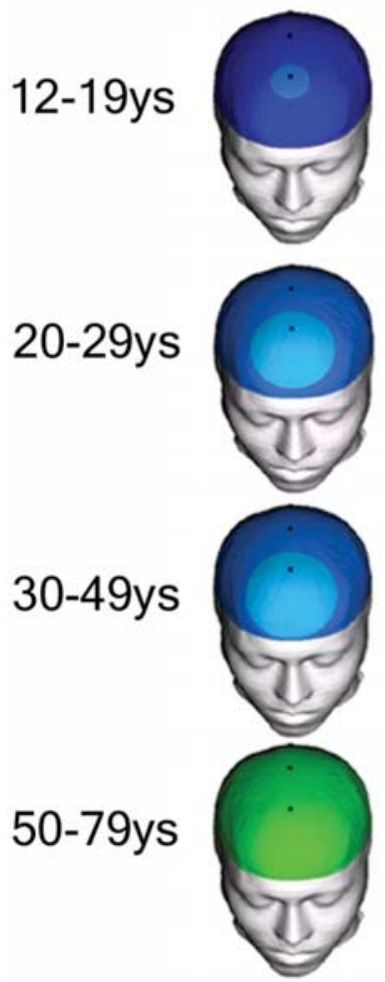

40-120ms

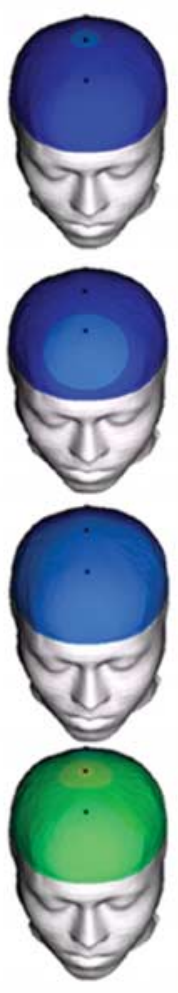

80-150ms
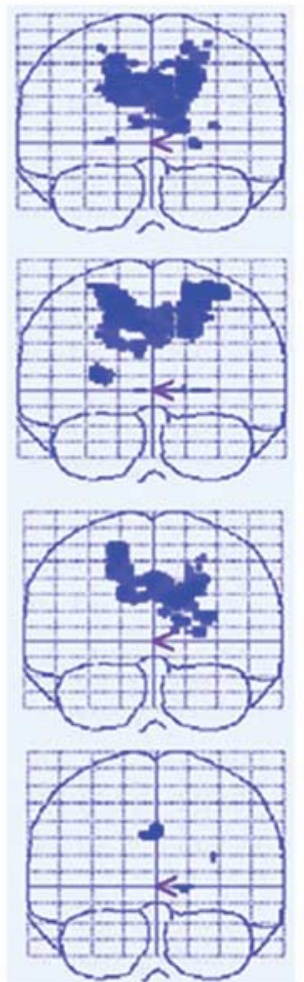

Medial Prefrontal Cortex activation
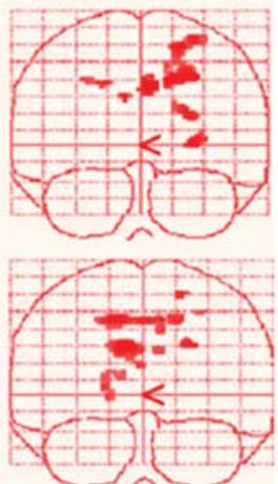

Increasing later responses to fear
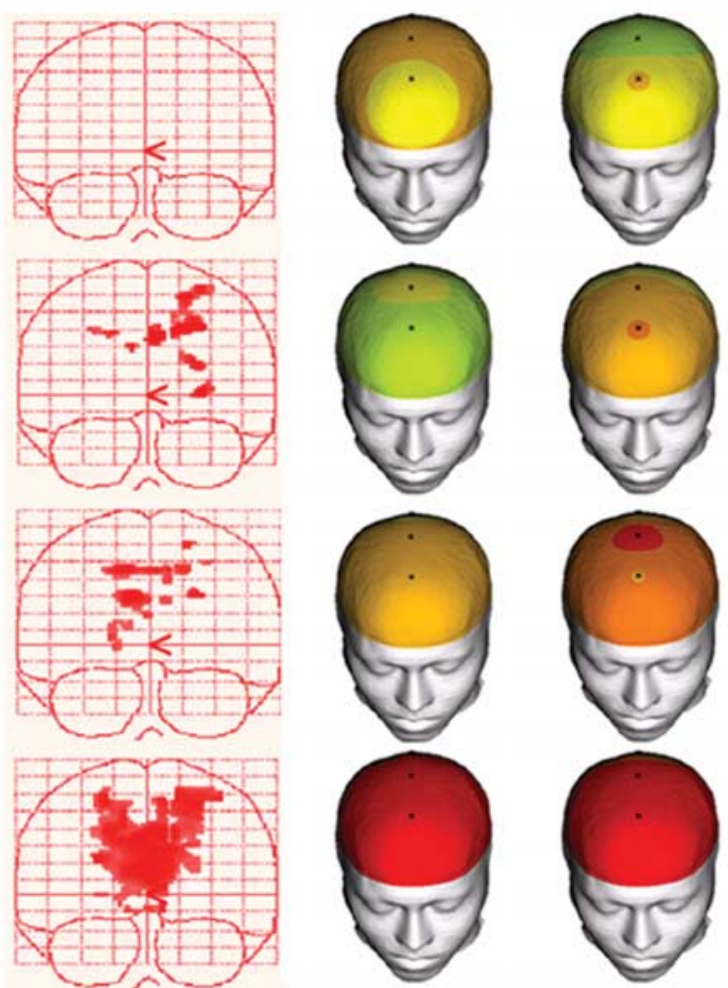

$180-280 \mathrm{~ms}$

$220-450 \mathrm{~ms}$

Figure 5. Changes in both ERP (over the time course $40-450$ ms after stimulus) and fMRI measures over age. Happiness relative to neutral showed a decrease in activity over the medial prefrontal region (shown in blue), which was most apparent for neural activity elicited during the early phase of processing (40-150 ms after stimulus). In response to fear, in contrast, there was an increase in activity over the medial prefrontal cortex with age (shown in red), which ERPs showed was most apparent for neural activity occurring in the later phase of the time course ( $180-450$ ms after stimulus). Coordinates for fMRI changes are shown in Table 1.

age was seen in both recognition and brain function. Recognition of negative emotion (fear) showed a significant decline as a function of increasing age, whereas recognition for positive emotion (happiness) increased, although these changes were small and limited by a ceiling effect for happiness. Fear elicited corresponding increases in medial prefrontal activation, apparent during the later (180-450 ms) controlled phase of processing (Patel and Azzam, 2005). In contrast, medial prefrontal activation to happiness attenuated over age, apparent in the early, automatic (within $150 \mathrm{~ms}$ ) phase of the time course. These changes indicate that processing resources are shifted from the automatic phase of stimulus appraisal toward a greater allocation during the controlled phase of stimulus evaluation. We propose that this shift in resources supports better selective control of reactions to negative, particularly threat-related stimulation and allows positive responses to proceed without restraint. Our observation that this shift in processing of negative versus positive emotion predicted better emotional wellbeing with older age supports the view that it contributes to an increasingly adaptive regulation of emotion.

The parallel dissociation in the time course of responses to fear and happiness over age may be interpreted in the context of previous electrophysiological studies. Salient emotional expressions, including both fear and happiness, have been found to modulate early fronto-central activity within the first 150-200 ms of processing (Eimer and Holmes, 2002; Esslen et al., 2004). These modulations are seen to reflect rapid and automatic top- down modulation of early perceptual appraisal of emotionally significant input, which precedes structural encoding (Adolphs, 2002; Eimer and Holmes, 2002). Fearful faces have been distinguished by later sustained activity persisting beyond $250 \mathrm{~ms}$, reflecting selective allocation of attention to emotionally relevant input (Eimer and Holmes, 2002; Williams et al., 2006a). Thus, the enhancement in extended voluntary attention to fear, but attenuation of early activation for positive input over age, suggests a shift in fronto-central activity that supports greater regulation of fear-related responses and allows automatic perceptual analysis of positive emotion to proceed in the absence of top-down modulation.

A locus of change in the medial prefrontal cortex is consistent with the purported role of this region in processes governing emotion, social, and self-regulation (Damasio, 1996). Indeed, activation in this region has been associated specifically with training in the upregulation and downregulation of emotional responses (Bonanno et al., 2004). Conversely, subcortical activation associated with processing fear and happiness was generally greater in younger subjects, in accord with previous neuroimaging findings (Iidaka et al., 2002; Gunning-Dixon et al., 2003; Mather et al., 2004). Although the subcortical amygdala has a key role in encoding the arousing aspects of emotion stimuli (Zald, 2003), it may be less crucial in older age, consistent with evidence that amygdala lesions occurring later in life cause only 
very minimal disruptions to automatic vigilance for fear (Dolan, 2002).

We propose that life experience and changing motivational goals may drive plasticity in the medial prefrontal brain systems to increase selective control over the balance of negative and positive emotion and with the consequence of improving emotional wellbeing. Twin study evidence shows that experience may indeed have a comparatively greater impact on emotional regulation in older age, although heritability has a greater impact on more transient changes in emotional function (Neiss and Almeida, 2004). The medial prefrontal cortex in particular has been implicated in rapid, experiencedependent emotional learning (Morris and Dolan, 2004), consistent with a primary role for this region in flexible updating of emotional learning as a function of experience.

Our finding that age-related changes in the structure of the medial prefrontal cortex did not contribute to emotional wellbeing indicates that experience and learning impact the function of this region in particular. Normal atrophy of the medial prefrontal region contributed only to decline in fear recognition, which was independent from the functional role of this region in better emotional stability. A more generalized and severe impairment in processing facial emotion may occur only with significant lesions of the medial prefrontal cortex (Hornak et al., 1996). Indeed, fear recognition remained substantially above chance level in older individuals, despite the reduction relative to younger years. The comparative preservation of the subcortical amygdala and basal ganglia structures is consistent with previous evidence (Calder et al., 2003; Grieve et al., 2005). Although preservation of these structures cannot account for opposing changes in emotion recognition, the stability of both the structure and function of subcortical regions may contribute to maintaining emotion recognition at above chance levels over age. This proposal accords with evidence that the amygdala is associated with relatively irreversible learned responses to biologically relevant stimuli (Morris and Dolan, 2004), which may rely on structural preservation.

The motivation to achieve better emotional control in older adults may come from the increasing awareness of one's mortality and the desire to maximize the meaningfulness of environmental events and input, over and above the need for acquisition (Carstensen and Lockenhoff, 2003). With repeated emotional experiences over the lifespan, humans may learn to be more selective about which input is likely to provide quality positive outcomes as opposed to cause distress. Consistent with this view, older adults are more selective about their social activities and tend to prioritize quality rather than quantity (Carstensen and Lockenhoff, 2003).

It has been demonstrated recently that neural reorganization can produce long-lasting changes in the electrical synapses of the mammalian brain (Landisman and Connors, 2005). Moreover, neuromodulator action plays a key role in plasticity and may be
Amygdala
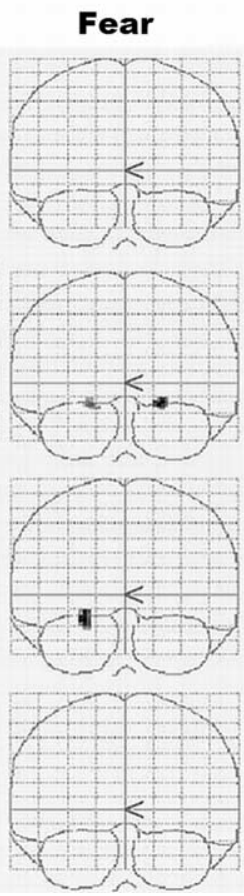

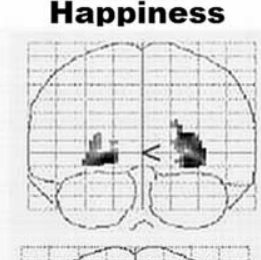

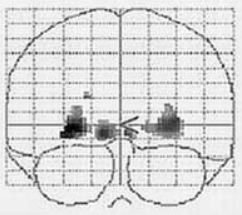

\section{Basal Ganglia}
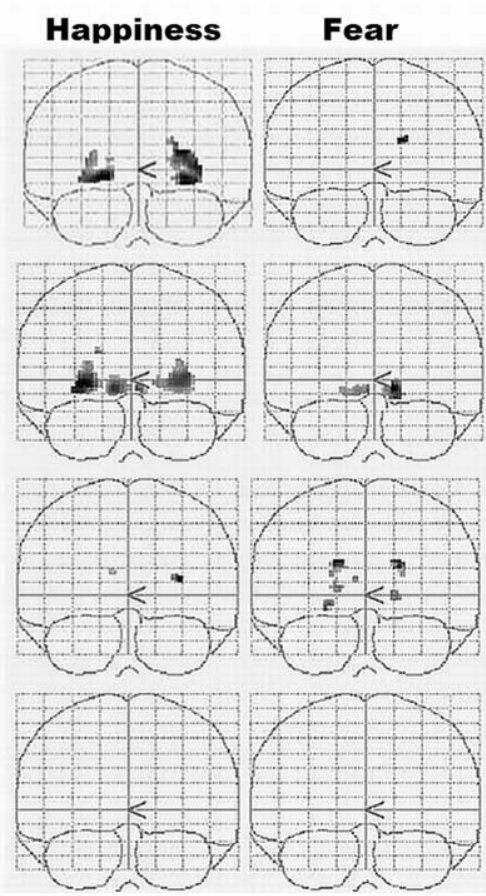

Figure 6. "Glass brain" statistical parameter maps showing the changes in subcortical amygdala and basal ganglia activity over in response to happiness and fear relative to neutral. These maps depict the different pattern of change that occurred ove

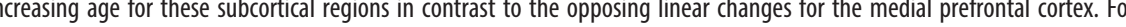
greater in the middle age groups ( $30-59$ years) compared with both the youngest (12-19years) and oldest (50-79years) groups for fear. Coordinates for these differences are shown in Table 1.

one mechanism by which changes in motivation and experience impact the emotion-related functions of the medial prefrontal cortex (Hamann et al., 1996; Dolan, 2002). Dopamine regulation occurs preferentially in the medial prefrontal cortex and has long been implicated in the regulation of emotion as well as motivation and affective behavior (Scheler, 2004). In primates, a loss of medial prefrontal dopamine receptor binding has nonetheless been associated with a corresponding increase in dopamine uptake (Moore et al., 2005). This finding is consistent with the proposal that cellular loss of receptors, attributable to age-related atrophy, may produce a compensatory mechanism of increased cell size and associated uptake (Stark and Pakkenberg, 2004). A similar mechanism of dopamine-related compensatory plasticity, driven by the environmental changes outlined above, may underlie the findings of our study. Investigation of genetic polymorphisms known to impact prefrontal dopamine regulation, such as catecholamine-O-methyltransferase (Meyer-Lindenberg et al., 2005), would provide a means to further explore the role of such a mechanism and the specific contributions of environmental and genetic influences in age-related improvements in emotional selectivity and stability.

An integrative model of emotional selectivity may have important clinical applications. Capacity for regulating negative versus positive emotion has been found to predict emotional health over the period after the September 11th terrorist attacks for those exposed to the trauma (Moore et al., 2005). Posttrauma reactions generally disrupt the regulation and response of the medial prefrontal cortex to salient signals of emotion (Bryant et 
al., 2005; Williams et al., 2006b). Thus, normal age-related variation in emotion regulation may be an important factor in determining which individuals have heightened vulnerability as opposed to resilience to the impact of trauma. Moreover, with predictions that one in three people will be $>60$ years old by 2150 (United Nations Secretariat, 1998), new interventions are also required to address the overwhelming increase in age-related cognitive impairment. Evidence that emotional brain function and behavior improves with age provides a platform from which new interventions might be developed to draw on strategies of emotional control to better cope with concomitant impairments in general cognitive function.

\section{References}

Adolphs R (2002) Neural systems for recognizing emotion. Curr Opin Neurobiol 12:169-177.

Ashburner J, Friston KJ (2000) Voxel-based morphometry: the methods. NeuroImage 1:805-821.

Ashley V, Vuilleumier P, Swick D (2004) Time course and specificity of event-related potentials to emotional expressions. NeuroReport 15:211-216.

Bonanno GA, Papa A, Lalande K, Westphal M, Coifman K (2004) The importance of being flexible: the ability to both enhance and suppress emotional expression predicts long-term adjustment. Psychol Sci 15:482-487.

Bryant RA, Felmingham KL, Kemp AH, Barton M, Rennie CJ, Gordon E, Williams LM (2005) Neural networks of information processing in posttraumatic stress disorder: a functional MRI study. Biol Psychiatry $58: 111-118$

Calder AJ, Keane J, Manly T, Sprengelmeyer R, Scott S, Nimmo-Smith I, Young AW (2003) Facial expression recognition across the adult life span. Neuropsychologia 41:195-202.

Carstensen LL, Lockenhoff CE (2003) Aging, emotion, and evolution: the bigger picture. Ann NY Acad Sci 1000:152-179.

Costa PT, McCrae RR (1992) Revised NEO Personality Inventory (NEOPI-R) and the Five Factor Inventory (NEO-FFI): professional manual. Odessa, FL: Psychological Assessment Resources.

Craik FIM, Salthouse TA, eds (2000) The handbook of aging and cognition, Ed 2. Mahwah, MJ: Elbaum.

Damasio AR (1996) The somatic marker hypothesis and the possible functions of the prefrontal cortex. Proc R Soc Lond B Biol Sci 351:1413-1420.

Dolan RJ (2002) Neuroscience and psychology: emotion, cognition and behavior. Science 298:1191-1194.

Eimer M, Holmes A (2002) An ERP study in the time course of emotional face processing. NeuroReport 13:427-431.

Esslen M, Pascual-Marqui RD, Hell D, Kochi K, Lehman D (2004) Brain areas and time course of emotional processing. NeuroImage 21:1189-1203.

George LK, Blazer DG, Winfield-Laird I, Leaf PJ, Fischbach RL (1988) Psychiatric disorders and mental health service use in later life: evidence from the Epidemiologic Catchment Area Program. In: Epidemiology and aging (Brody J, Maddox G, eds), pp 189-219. New York: Springer.

Good CD, Johnsrude I, Ashburner J, Henson RNA, Friston KJ, Frackowiak RSJ (2001) A voxel-based morphometric study of aging in 465 normal adult human brains. NeuroImage 14:21-36.

Gordon E, Cooper N, Rennie C, Hermens D, Williams LM (2005) Integrative neuroscience: the role of a standardized database. Clin EEG Neurosci 36:64-75.

Grieve SM, Clark CR, Williams LM, Peduto AS, Gordon E (2005) Preservation of limbic and paralimbic structures in aging. Hum Brain Map 25:391-401.

Gross JJ, Carstensen LL, Pasupathi M, Tsai J, Gotestam Skorpen C, Hsu AY (1997) Emotion and aging: experience, expression, and control. Psychol Aging 12:590-599.

Gunning-Dixon FM, Gur RC, Perkins AC, Schroeder L, Turner T, Turetsky BI, Chan RM, Loughead JW, Alsop DC, Maldijan J, Gur RE (2003) Agerelated differences in brain activation during emotional face processing. Neurobiol Aging 24:285-295.

Hamann SB, Stefanacci L, Squire LR, Adolphs R, Tranel D, Damasio H, Damasio A (1996) Recognizing facial emotion. Nature 379:497.

Helson R, Wink P (1987) Two conceptions of maturity examined in the findings of a longitudinal study. J Pers Soc Psychol 53:531-541.

Hornak J, Rolls ET, Wade D (1996) Face and voice expression identification in patients with emotional and behavioral changes following ventral frontal lobe damage. Neuropsychologia 34:247-261.

Iidaka T, Okada T, Murata T, Omori M, Kosaka H, Sadato N, Yonekura Y (2002) Age-related differences in the medial temporal lobe responses to emotional faces as revealed by fMRI. Hippocampus 12:352-362.

Jeffreys DA (1989) A face-responsive potential recorded from the human scalp. Exp Brain Res 78:193-202.

LaBar KS, Gitelman DR, Mesulam MM, Parrish TB (2001) Impact of signalto-noise on functional MRI of the human amygdala. NeuroReport 12:3461-3464.

Lachman ME, Bertrand RM (2001) Personality and the self in midlife. In: Handbook of midlife development (Lachman ME, ed). New York: Wiley.

Landisman CE, Connors BW (2005) Long-term modulation of electrical synapses in the mammalian thalamus. Science 310:1809-1813.

Malatesta CZ, Izard C, Culver C, Nicolich M (1987) Emotion communication skills in young, middle-aged, and older women. Psychol Aging 2:193-203.

Mather M, Canli T, English T, Whitfield S, Wais P, Ochsner K, Gabrieli JDE, Carstensen LL (2004) Amygdala responses to emotionally valenced stimuli in older and younger adults. Psychol Sci 15:259-263.

Meyer-Lindenberg A, Kohn PD, Kolachana B, Kippenhan S, McInerney-Leo A, Nussbaum R, Weinberger DR, Berman KF (2005) Midbrain dopamine and prefrontal function in humans: interaction and modulation by COMT genotype. Nat Neurosci 8:594-596.

Moore TL, Schettler SP, Killiany RJ, Herndon JG, Luebke JI, Moss MB, Rosene DL (2005) Cognitive impairment in aged rhesus monkeys associated with monoamine receptors in the prefrontal cortex. Behav Brain Res 160:208-221.

Moreno C, Borod JC, Welkowitz J, Alpert M (1993) The perception of facial emotion across the adult life-span. Dev Neuropsychol 9:305-314.

Morris JS, Dolan RJ (2004) Dissociable amygdala and orbitofrontal responses during reversal fear conditioning. NeuroImage 22:372-380.

Morris JS, Frith CD, Perrett DI, Rowland D, Young AW, Calder AJ, Dolan RJ (1996) A differential neural response in the human amygdala to fearful and happy facial expressions. Nature 383:812-815.

Mroczek DK, Spiro III RA (2003) Modeling intraindividual change in personality traits: findings from the normative aging study. J Gerontol 58B:153-165.

Neiss M, Almeida DM (2004) Age differences in the heritability of mean and intraindividual variation of psychological distress. Gerontology 50:22-27.

Oschsner KN, Ray RD, Cooper JC, Robertson ER, Chopra S, Gabrieli JDE, Gross JJ (2004) For better or worse: neural systems supporting the cognitive down- and up-regulation of negative emotion. NeuroImage 23:483-499.

Parrish TB, Gitelman DR, LaBar KS, Mesulam M-M (2000) Impact of signal-to-noise on functional MRI. Magn Reson Med 44:925-932.

Patel SH, Azzam PN (2005) Characterization of N200 and P300: Selected studies of the event-related potential. Int J Med Sci 2:147-154.

Phan KL, Wager T, Taylor SF, Liberzon I (2002) Functional neuroanatomy of emotion: a meta-analysis of emotion activation studies in PET and fMRI. NeuroImage 16:331-348.

Phillips LH, Allen R (2004) Adult aging and the perceived intensity of emotions in faces and stories. Aging Clin Exp Res 16:190-199.

Roberts B, Caspi A (2003) The cumulative continuity model of personality development: striking a balance between continuity and change in personality traits across the life course. In: Understanding human development: dialogs with lifespan psychology (Staudinger UM, Lindenberger U, eds), pp 183-214. New York: Kluwer.

Scheler G (2004) Regulation of neuromodulator receptor efficacy - implications for whole-neuron and synaptic plasticity. Prog Neurobiol 72:399-415.

Stark AK, Pakkenberg B (2004) Histological changes of the dopaminergic nigrostriatal system in aging. Cell Tissue Res 318:81-92.

Streit M, Wöwler W, Brinkmeyer J, Ihl R, Gaebel W (2000) Electrophysiological correlates of emotional and structural face processing in humans. Neurosci Lett 278:13-16.

Tzourio-Mazoyer N, Landeau B, Papathanassiou D, Crivello F, Etard O, Delcroix N, Mazoyer B, Joliot M (2002) Automated anatomical labeling of activations in SPM using a macroscopic anatomical parcellation of the MNI MRI single-subject brain. NeuroImage 15:273-289. 
United Nations Secretariat (1998) World population prospects, Vol II, Sex and age. New York: The Population Division, Department of Economic and Social Affairs.

Whittington JE, Huppert FA (1998) Neuroticism, psychiatric symptoms and life events. Pers Individ Dif 24:97-107.

Wild B, Erb M, Bartels M (2001) Are emotions contagious? Evoked emotions while viewing emotionally expressive faces: quality, quantity, time course and gender differences. Psychiatry Res 102:109-124.

Williams LM, Phillips ML, Brammer MJ, Skerrett D, Lagopoulos J, Rennie C, Bahramali H, Olivieri G, David AS, Peduto A, Gordon E (2001) Arousal dissociates amygdala and hippocampal fear responses: evidence from simultaneous fMRI and skin conductance recording, NeuroImage 14:1070-1079.
Williams LM, Das P, Liddell BJ, Olivieri G, Peduto AS, Brammer MJ, Gordon E (2005) BOLD, sweat and fears: functional BOLD, sweat and fears: fMRI and skin conductance distinguish facial fear signals. NeuroReport 19:49-52.

Williams LM, Palmer DM, Liddell BJ, Song L, Gordon E (2006a) The "when" and "where" of perceiving signals of threat and non-threat. NeuroImage, 31:458-467.

Williams LM, Kemp AH, Felmingham K, Barton M, Olivieri G, Peduto AS, Gordon E, Bryant RA (2006b) Trauma modulates amygdala and medial prefrontal responses to consciously attended fear. NeuroImage 29:347-357.

Zald DH (2003) The human amygdala and the emotional evaluation of sensory stimuli. Brain Res Rev 41:88-123. 